\title{
Does collective consciousness have a role in radiation mass exposures and epidemics?
}

\author{
Indraganti PK*, Namita I and Khanna A \\ Drug Repurposing and Translational Research Lab, Institute of Nuclear Medicine and Allied Sciences, Brig SK Majumdar Road, Timarpur, Delhi-110054, India
}

\begin{abstract}
Every event receives people's attention either locally or globally. Major events (like radiation accidents, epidemics and pandemics) affecting a large number of people often receive world's attention which peaks soon after the event and subsides gradually. However, the dynamics of cause-effect in major events is not well understood and it is not clear if the focused attention of normal population impacts the well-being of the exposed victims and how different groups interact with each other. The idea of panpsychism and hierarchical consciousness suggests that consciousness prevails beyond an individual and operates at many levels like family, community, state, territories, nations, and world consciousness. In this perspective, based on the idea of hierarchical consciousness, the impact of collective consciousness in major events like mass radiation exposures or epidemics has been discussed. It is being suggested that higher consciousness (focused attention of a large number of people) impacts (which can be either detrimental or constructive) the well-being of exposed population. The possible role of quantum entanglement in exerting the impact of the focused thoughts of a large number of people (collective consciousness) has also been discussed.
\end{abstract}

\section{The need for effective management after mass radiation overexposures or epidemics and pandemics}

Nuclear, chemical, biological warfare or epidemics and pandemics results in exposure of a large number of human populations with catastrophicimmediate and long-term consequences [1,2]. The mortality encountered during such situations is often related to the absorbed dose of the radiation (in case of a nuclear accident) or the virulence of the causative pathogen (in case of epidemics and pandemics). Factors like fear, stress in general are known to further aggravate the overall mortality or well-being through psycho-immunological and psycho-physiological routes [3]. However, it is interesting to delve upon other possible factors which might be contributing to the overall mortality or other outcomes during mass exposure scenarios as better understanding can be expected to facilitate better management. The effect of the post-event thoughts (focused attention) of bystanders or normal (unexposed) population (the adjoining community's, cities, adjacent countries and rest of the world) on overall mortality, if any, has not been deciphered properly. In this article an attempt has been made to discuss the following questions:

1. Does the focused attention of the unexposed normal population (like communities, territories, states, adjacent countries, nations) influence the exposed population?

2. Does collective consciousness drive the occurrence of the events?

Before the role of focused thoughts of normal population (increased layers of consciousness) on overall mortality during mass exposures is discussed, first, it is important to understand the dynamics of a group.

\section{Life has been optimized for survival}

Organisms have evolved both progressively as well as regressively for optimizing the survival of a species rather than to act most efficiently or for realizing objective reality of the world [4]. At an individual level, the system takes a plethora of decisions for maximizing its survival, however, when it comes to a group of animals, decisions are taken to maximize the survival of the group rather than individuals comprising it. For example, when cells receive damage to DNA, DNA damage response gets into operation and tries to repair the damage [5]. However, when it becomes irreparable then the cells initiate apoptosis in response to both intrinsic and extrinsic signals [6]. In case of radiation overexposure, a large number of cells from radiosensitive tissues (like bone marrow, spleen, thymus etc.) are removed through apoptotic pathway to ensure that cells with compromised genome are eliminated from the system which is advantageous for long-term survival of the species as such cells with compromised genome can transform to tumorigenic [7]. The same logic can be extended to organisms surviving as colonies and can be expected to have a strategy ensuring the overall survival. In case of colonizing bacteria, which is made up of individual bacterium and act as one unit, the fate of each individual bacterium should be under the control of the colony. In fact, the bacterium should be undergoing apoptosis or similar mechanisms leading to self- killing, provided its elimination offers advantage to the overall survival of the community. As a matter of fact, E. coli which is a colonizing bacterium has requisite cellular machinery to execute apoptosis under certain conditions. It has been reported that E. coli has maz E (which encodes for an antitoxin) and maz F (which codes for a toxin) system along with Rec A system which can initiate apoptosis in response to heavy DNA damage [8,9]. In

${ }^{*}$ Correspondence to: Prem Kumar Indraganti, Drug Repurposing and Translational Research Lab, Institute of Nuclear Medicine and Allied Sciences, Brig SK Majumdar Road, Timarpur, Delhi-110054, India, Tel: 91-11-23905301/919958456999; E-Mail: prem@inmas.drdo.in/prem_indra@yahoo.co.in

Key words: consciousness, collective consciousness, hierarchical consciousness, epidemics, knowledge burden, radiation accidents, global consciousness project, quantum entanglement, reality

Received: March 02, 2020; Accepted: March 16, 2020; Published: March 23 2020 
fact, maz E/F system inhibits Rec A mediated apoptosis through EDF which is an external factor which is released by other bacteria in the community through quorum sensing. Basically, the colony is controlling the cellular activities and fate of the individual. In this case both Rec A and maz E/F are cell death inducers. maz E/F in the presence of EDF, induces apoptosis and also inhibit Rec A pathways [8]. These studies clearly suggest that a group as an entity controls the behavior and fate of the individuals comprising the group. This logic can be extended to mammals including humans which are social and survive in groups. However, it is interesting to decipher the means through which a group (family, community, state, nations etc.) connects and executes as a unit.

\section{Does consciousness connect a group?}

A number of animal species which are social in nature survive as a unit with well-defined division of labour (like ants, bees, wasps, dolphins, chimpanzees etc.) which maximizes the overall survival of the species [10-12]. An important and long-standing question which intrigued both researchers and philosophers is what connects a group and makes them act like a unit. Normally, an individual with trillions of cells divided into tissues, organs and systems are interconnected and function essentially based on communication of information (signalling pathways). Similarly, a group of people, like family, society, community, regions, states etc. also function as one unit with an ultimate goal to safeguard the overall survival. Though a group is not a physically connected system, nevertheless it functions as a unit essentially based on communication involving exchange of information and possession of a collective viewpoint. Several researchers have suggested that among the non-physical factors which connect a group of individuals include collective consciousness. In fact, sociologist like Émile Durkheim sees the collective conscience as a key non-material social fact. The collective conscience is "the totality of beliefs and sentiments common to average citizens of the same society" [13]. Consciousness, a state of being able to subjectively experience something has been a subject of intense debate. Though a large number of theories have been proposed for addressing a number of aspects related to consciousness, but a general consensus is still eluding. Panpsychism, a dominant theory in the field of consciousness during 17th century, which has recently witnessed a renewed interest, argues that every entity has some consciousness $[14,15]$. Based on the idea of panpsychism, recently we proposed that hierarchical consciousness is at play and suggested the existence of a number of conscious layers (like atoms, molecules, cells, tissue, organs, systems, organism) with the individual consciousness forming the top most layer (from an individual point of view) [16]. Within an individual, every aspect of biology is controlled and a given conscious entity is directly under the control of an immediate higher level (for example changes at atomic level is brought about by the molecules and changes at cellular level is in turn brought about by the tissues (collection of cells) [16]. With the assumption of layered consciousness, it can be stated that consciousness possesses the capacity to extend beyond an individual and family, community, state and so on and may form the higher levels of consciousness. As of now a majority of the discussions on collective consciousness is qualitative in nature and has not been properly deciphered or quantified. The global consciousness project was initiated in 1997 and using random number generators (RNG) which produce unpredictable sequences of zeros and ones but when a global event synchronizes a large number of people then the RNGS deviate from being random to structured indicating the prevalence of collective consciousness $[17,18]$. In spite of the debate over the methodology adopted and interpretation of the data, arguably this has been the most scientific attempt yet and indicates the existence of global consciousness [19]. In fact, global consciousness project and a number of other studies, evaluated the outcome of coherent group events (like cricket, football world cup, major world events like 09-11-2001 terrorist attack) and suggested that it in fact, impacts the outcome [20].

The above discussion suggests that consciousness extends beyond an individual and the higher order consciousness (collective consciousness) may influence the lower layers. This logic will be extended to a radiation mass exposure scenario to unravel the impact of higher consciousness (like communities, territories, states, adjacent countries, nations) on the exposed population.

\section{Consciousness and manifestation of radiation induced damage-The dynamics}

Increase in the number of hierarchical consciousness is inversely related to the radio sensitivity of the organisms [16]. A typical colonizing bacteria, like E. coli, which is connected to the other bacteria of the colony through quorum sensing, seems to be under the regulation of a lesser number of conscious levels (atomic, molecular, supra molecular, organelle, cellular, community) when compared to a cell (like a lymphocyte) in a mammalian system. As signalling and communication is the basis for controlled functioning of an organism, the topmost layers would be connected to lower layers through different signalling pathways. As it can be expected, more pathways mean anything which is disturbing a particular pathway would have more impact because a single pathway controls a number of different systems. The complexity (multiple pathways controlling the whole system in a coordinate fashion to optimize the overall survival of the organism) that is associated with multi-cellular organism improves the chances to maintain the internal homeostasis in spite of changing external and internal environments. It means, as the consciousness increases, the threshold for the damage also increases but once it is compromised then it would have serious impact on overall health of the organism because many lower layers are immediately under its control. In fact, it seems to be the case at least in the event of radiation over exposure scenarios. For example, a mouse exposed to a wholebody irradiation of 2 gray (Gy) (sub lethal dose) would not lead to any lethality and the damage incurred to different cell types gets recovered effectively. It does not involve collapse of any system (hematopoieic form of acute radiation syndrome (HP-ARS) or gastrointestinal form of acute radiation syndrome (GI-ARS) or cerebrovascular form of acute radiation syndrome (CNS). However, a dose of 8-10 Gy (lethal dose) would result in collapse of the hematopoietic system and death occurs essentially in 14-15 days. An exposure of supra lethal dose (>12 Gy) would result in the collapse of the GI system and death essentially happens within 9 days and an exposure of more than 25 Gy would result in cerebrovascular syndrome and death ensues much faster and in the matter of a few days the animals succumb to radiation effects. Similar correlation can be drawn in case of humans also [21,22]. As suggested, based on hierarchical consciousness, a layer superior to an individual (like family, community, regions, state, etc.) when compromised (it is considered when negative viewpoint is prevailing in large number of people) would severely impact the well-being of lower layers. It means a group of individuals exposed to a dose of radiation would impact the individuals of the group more than when an individual of a group is singly exposed to the same dose. In fact, in 1963 Hahn and Howland showed that caging influences the radiation induced lethality and demonstrated that rats exposed in groups are more radiosensitive when compared to rats exposed singly indicating that a group influences the mortality in individuals of the group [23]. Based on this discussion, it can 
be suggested that focused attention or viewpoint of normal bystander population (adjacent communities, states) may impact the outcome of the exposed victims. The effect can be positive (helps in recovery and restitution of normalcy) or deteriorating (increases the mortality). It means the mortality after nuclear accidents or epidemics depends not only on the absorbed radiation dose or virulence of the pathogen but also on the viewpoint that a population holds. The viewpoint of the population depends upon the prior information. In view of the technological advancement and the social media, information is readily and easily accessible (to large extent fake information) and is obvious that more the spread of negative information more are the chances that collective consciousness would hold negative thoughts and impact negatively [24]. However, this warrants an explanation on how the focused thoughts of a large number of people (collective consciousness) manifest.

\section{Consciousness and reality}

Focused thoughts of a large number of people can be expected to influence the outcome, if it is considered that somehow the information (focused thoughts or viewpoint) gets transferred thereby making it a reality. Here we used a scenario presented by Roger Nelson in his publication to argue that focused thoughts can transmit and manifest as a reality. Using a single event which had an impact at global level (the 09-11-2001 terrorist attack) recently, he suggested that the cumulative deviation (indicative of non-randomness) begins as early as a day before the planes crashed into the world trade centre [25], suggesting that somehow the (for convenience sake free will is considered to be in operation) consciousness was sensing the upcoming event. Here, two different scenarios are presented to address the probable relationship between the event [9-11] and change in cumulative deviation that was observed prior to the event. Firstly, the global consciousness is somehow sensing the upcoming event as suggested by Roger Nelson, global consciousness might manifest presentiments of the future $[26,27]$. Secondly, the event is driven by the collective consciousness and the deviation observed prior to the event indicates that when a large number of people focus (one opinion) on one thing then it becomes the reality (a major event). Considering that a small group of people, the terrorists, involved in the attack, it is difficult to ascribe their focused thoughts as responsible for the deviation observed prior to the event as coherence among small number of people is associated with small effects [26]. However, this conundrum to some extent can be solved by considering that a significant fraction of collective consciousness (public sentiments across a region or a globe which is much more than the focused attention of the small group of terrorists who carried out the attack) was having focused (consciously or subconsciously) thoughts about an attack and the terrorist attack witnessed on 9-11-2001 was the resultant of such a large scale focused thoughts. This argument fundamentally considers that focused thoughts at individual or mass levels can influence the outcome (this argument does not fall into the realms of mysticism if one considers hierarchical consciousness and quantum entanglement in action). Even though at present we do not have a scientifically verifiable rational explanation on how focused thoughts influence the reality (outcome) but efforts will be made to put forward this argument in a rational way. In our lifetime, many of us (it is actually applicable to all) must have experienced a situation when we thought of something or someone and at times it comes true or we encounter the person whom we were thinking about. The reason why it works at times and not all the time, is probably due to the final information one carries (the final thought about any given situation). It will become reality when you just have one thought (for example that you meet the person) and often it comes true when one thinks about meeting a friend and ceases to rethink or reanalyse (hence the final thought is seeing the friend). In fact, this can be tested and individually verified by the readers of the article. Interestingly, a retrospective analysis is the only possibility as prospectively assessing is a problem because of the knowledge burden. As any conscious efforts would involve rational analysis which in turn depends upon the prior knowledge (the knowledge burden) which would make you hold a viewpoint accordingly (we just cannot conjure things to happen). If this argument is given serious consideration then it is likely the reason why we perceive a cat as a cat is because of the prior knowledge (the viewpoint one holds) and if you can hold a different view and when that becomes the only view, then one should end up in seeing it differently. Though it is not clear, but it is interesting to note why do we get such momentary thoughts which we don't delve in any further (this definitely complicates the idea of free will). In fact, a number of practices like meditation, Yoga, and any method which achieves focus or concentration, just ensures one to have a single thought and in fact a number of earlier studies with focused groups suggested that it impacts a variety of phenomenon and supports this assumption [27]. It would be easier if the idea is explained using an example. Schrödinger's cat is a thought experiment proposed by Erwin Schrodinger to illustrate the weirdness of the state of quantum superposition [28]. It presents a hypothetical cat which is both alive and dead at the same time until an observation is made. This superposition roots from the fact that the observer does not have any knowledge (viewpoint) about the status of the cat so he cannot hold one view (cat either dead or alive). However, if somehow, the observer can hold just one view (consciously or sub-consciously) then it would become the reality and whatever the viewpoint he is holding will actually prevail. As it can be seen, the critical part is to hold just one view.

\section{How the global consciousness impacts outcome-The quantum entanglement}

Quantum entanglement is the physical phenomenon in which the quantum state of a pair or group of particles, emanated from the same process, have to be described with reference to each other, even though the individual objects may be spatially separated [29]. Considering that singularity was the initial entity which has given rise to entire cosmos after the big bang, it can be argued that the entire cosmos is connected in a way irrespective of distance. This also means that we may be influencing each other and every event (every event irrespective of whether a minor or a major event) that is happening. In the context of mass exposures (either epidemics or ionizing radiation) we influence each other and the outcome.

\section{Conclusion}

The above discussion essentially suggests that the world is an interconnected system which can be influenced in a number of ways. The consciousness seems to be the underlying phenomenon which is controlling the dynamics of a group. In case of a major event (epidemics or radiation over exposure) the higher consciousness (adjacent communities, cities, states, nation, world) may influence the exposed population based on the viewpoint or focused thoughts it holds, which in turn is dependent upon the information available. The focused viewpoint may be manifesting through quantum entanglement. Though, radiation accidents have been considered in this discussion, but it should be applicable to all the events including epidemics, riots, terrorist attacks, games, elections etc. 


\section{References}

1. Institute of Medicine (1986) The medical implications of nuclear war. Washington, DC: The National Academies Press.

2. Fineberg HV (2014) Pandemic preparedness and response--lessons from the H1N1 influenza of 2009. N Engl J Med 370: 1335-1342. [Crossref]

3. O'Leary A, Jalloh MF, Neria Y (2018) Fear and culture: contextualising mental health impact of the 2014-2016 Ebola epidemic in West Africa. BMJ Glob Health 3: e000924. [Crossref]

4. Hoffman D (2019) The case against reality - Why evolution hid the truth from our eyes. W. W. Norton \& Company (30 July 2019).

5. Jackson SP, Bartek J (2009) The DNA-damage response in human biology and disease. Nature 461: 1071-1078. [Crossref]

6. Roos WP, Thomas AD, Kaina B (2016) DNA damage and the balance between survival and death in cancer biology. Nat Rev Cancer 16: 20-33. [Crossref]

7. Renehan AG, Booth C, Potten CS (2001) What is apoptosis, and why is it important? $B M J$ 322: 1536-1538. [Crossref]

8. Erental A, Sharon I, Engelberg-Kulka H (2012) Two programmed cell death systems in Escherichia coli: an apoptotic-like death is inhibited by the mazEF-mediated death pathway. PLoS Biol 10: e1001281. [Crossref]

9. Robinson R (2012) In E. coli, interrupting one death pathway leads you down another. PLoS Biol 10: e1001278. [Crossref]

10. Cooper GA, West SA (2018) Division of labour and the evolution of extreme specialization. Nat Ecol Evol 2: 1161-1167. [Crossref]

11. Highfill L, Frick E (2016) Social Mammals. In: Zeigler-Hill V., Shackelford T. (eds) Encyclopedia of Personality and Individual Differences. Springer, Cham.

12. Simpson, George (1993) in Durkheim, Emile "The Division of Labour in Society" The Free Press, New York. pp. ix.

13. Durkheim, Emile (1997) The Division of Labour in Society. Trans. W. D. Halls, intro. Lewis A. Coser. New York: Free Press. pp. 39, 60, 108.
14. Morch HH (2017) Is matter conscious? Why the central problem in neuroscience mirrored in physics. Nautilus 6 .

15. Bailey A (1922) The consciousness of the atom. A series of lectures delivered in New York City, pp: 1921-1922.

16. Indraganti PK, Namita I, Khanna A (2019) Consciousness: A Possible Gateway for Protection Against Radiation Induced Damage. Austin Biochem 4: 1024.

17. Nelson RD, Radin DI, Shoup R, Bancel PA (2002) Correlations of Continuous Random Data with Major World Events. Found of Phys Lett 15: 537-550.

18. Global consciousness project (http://noosphere.princeton.edu).

19. Bancel PA, Nelson RD (2008) The GCP event experiment: Design, analytical methods, results. J Sci Explor 22: 309-333.

20. Nelson RD (2019) Connected: The emergence of global consciousness. ICRL Press, Princeton, NJ.

21. Williams JP, Brown SL, Georges GE et al (2010) Animal models for medical countermeasures to radiation exposure. Radiat Res 173: 557-578.

22. Macià I Garau M, Lucas Calduch A, López EC (2011) Radiobiology of the acute radiation syndrome. Rep Pract Oncol Radiother 16: 123-130.

23. Hahn EW, Howland JW (1963) Modification of irradiation response of female rats by population density. Radiat Res 19: 676-681.

24. Collinson S, Khan K, Jane M, Heffernan JM (2015) The effects of media reports on disease spread and important public health measurements. PLoS One 10: e0141423.

25. Nelson RD (2019) Evoked potentials and GCP event data. J Sci Explor 12.

26. Radin DI (2004) Electrodermal presentiments of future emotions. J Sci Explor 18: 253273.

27. Ball J (2011) Collective consciousness and meditation: Are we all interconnected by an underlying field? Huffpost 16: 02.

28. Ball P (2018) Real-life schrödinger's cats probe the boundary of the quantum world Quantamagazine. June 25, 2018.

29. Wilczek F (2016) Entanglement made simple. Quantamagazine April 28, 2016.

Copyright: $@ 2020$ Indraganti PK. This is an open-access article distributed under the terms of the Creative Commons Attribution License, which permits unrestricted use, distribution, and reproduction in any medium, provided the original author and source are credited. 